\title{
Fibroblasts and myofibroblasts in wound healing
}

This article was published in the following Dove Press journal:

Clinical, Cosmetic and Investigational Dermatology

6 November 2014

Number of times this article has been viewed

\author{
Ian A Darby' \\ Betty Laverdet ${ }^{2}$ \\ Frédéric Bonté 3 \\ Alexis Desmoulière ${ }^{2}$ \\ 'School of Medical Sciences, RMIT \\ University, Melbourne, VIC, Australia; \\ ${ }^{2}$ Department of Physiology and EA \\ 6309, FR 3503, Faculties of Medicine \\ and Pharmacy, University of Limoges, \\ Limoges, France; ${ }^{2}$ LVMH Recherche, \\ Saint Jean de Braye, France
}

\begin{abstract}
Myo)fibroblasts are key players for maintaining skin homeostasis and for orchestrating physiological tissue repair. (Myo)fibroblasts are embedded in a sophisticated extracellular matrix (ECM) that they secrete, and a complex and interactive dialogue exists between (myo)fibroblasts and their microenvironment. In addition to the secretion of the ECM, (myo)fibroblasts, by secreting matrix metalloproteinases and tissue inhibitors of metalloproteinases, are able to remodel this ECM. (Myo)fibroblasts and their microenvironment form an evolving network during tissue repair, with reciprocal actions leading to cell differentiation, proliferation, quiescence, or apoptosis, and actions on growth factor bioavailability by binding, sequestration, and activation. In addition, the (myo)fibroblast phenotype is regulated by mechanical stresses to which they are subjected and thus by mechanical signaling. In pathological situations (excessive scarring or fibrosis), or during aging, this dialogue between the (myo)fibroblasts and their microenvironment may be altered or disrupted, leading to repair defects or to injuries with damaged and/or cosmetic skin alterations such as wrinkle development. The intimate dialogue between the (myo)fibroblasts and their microenvironment therefore represents a fascinating domain that must be better understood in order not only to characterize new therapeutic targets and drugs able to prevent or treat pathological developments but also to interfere with skin alterations observed during normal aging or premature aging induced by a deleterious environment.
\end{abstract}

Keywords: myofibroblast, fibroblast, $\alpha$-smooth muscle actin, mechanical signaling, fibrosis, scarring

\section{Introduction to the myofibroblastic phenotype}

Myofibroblasts were first described in healing skin wounds, where it was hypothesized that they were responsible for the phenomenon of wound contraction. ${ }^{1}$ Since then, cells morphologically similar to myofibroblasts have been described in many tissues, predominantly in pathological states where their sustained presence is generally a marker of fibrosis and scarring. ${ }^{2}$

Early studies identified myofibroblasts on the basis of their ultrastructural morphology, with prominent microfilament bundles in their cytoplasm distinguishing them from "normal" quiescent tissue fibroblasts. Myofibroblasts also possessed fibronexus junctions between cells and the surrounding extracellular matrix (ECM), thus in some ways appearing to share some of the morphological characteristics of smooth muscle (SM) cells. ${ }^{3}$

Many tissues and pathologies have been described in which myofibroblasts have been identified, including hypertrophic and keloid scars in the skin, fibrotic liver as seen in liver cirrhosis and other liver pathologies, renal fibrosis, and idiopathic
Correspondence: Alexis Desmoulière Pharmacy, University of Limoges,

2 Rue du Dr Marcland,

87025 Limoges Cedex, France

Tel +33555435873

Fax +33555435950

Email alexis.desmouliere@unilim.fr 
pulmonary fibrosis. ${ }^{4}$ More recently, cells with phenotypic features of myofibroblasts have also been found in and around a number of epithelial tumors, where they have been termed cancer-associated fibroblasts or stromal myofibroblasts. ${ }^{5-7}$ The role of myofibroblasts in driving fibrotic diseases, and the recent finding that cancer-associated myofibroblasts likely influence tumor growth and correlate with poor clinical prognosis, has increased our interest in their cellular origins, their regulation, and their role in repair and disease. ${ }^{8}$

After early studies that defined myofibroblasts on the basis of their ultrastructural morphology, later research using antibodies and immunohistochemistry resulted in myofibroblasts and their microenvironment being more clearly defined. ${ }^{9}$ It is now accepted that myofibroblasts go through a precursor stage of expressing large stress fibers that are not seen in quiescent fibroblasts, prominent bundles of microfilaments that permit some contraction and pre-stressing and remodeling of the surrounding ECM. ${ }^{10}$ Later, fully differentiated myofibroblasts show expression of the usually SM-specific cytoskeletal protein, $\alpha-S M$ actin, which is now often used to define the myofibroblast phenotype. ${ }^{9,11}$

The presence of a splice variant form of fibronectin (ED-A fibronectin) in the microenvironment adjacent to the myofibroblast is also a defining feature and appears to be required for their differentiation. ${ }^{12}$ De novo expression of osteoblast $(\mathrm{OB})$ cadherin has also been reported to be found on the surface of differentiated myofibroblasts, and is not seen on $\alpha$-SM actin-negative fibroblasts. ${ }^{13}$

The other defining feature of myofibroblasts is that they fail to express the full repertoire of SM cell markers, allowing myofibroblasts to be distinguished from SM cells. Specifically, myofibroblasts in most cases are negative for SM cell markers such as SM myosin heavy chain, ${ }^{14}$ n-caldesmon, ${ }^{15}$ and smoothelin. ${ }^{16}$ Desmin has also been used as a negative marker of myofibroblasts. Generally, SM cells express desmin and vimentin as well as SM myosin, while myofibroblasts express only vimentin. However, some situations have been reported in the literature where myofibroblasts in some pathologies have been found to be desmin positive. ${ }^{17}$ Distinguishing myofibroblasts from pericytes is perhaps more problematic since pericytes can closely resemble myofibroblasts in being $\alpha$-SM actin positive, vimentin and desmin positive, but SM myosin negative..$^{15,18}$ Indeed, pericytes may in some cases be a source of myofibroblasts in some conditions, including wound repair, where myofibroblasts may represent a pericyte that has lost some phenotypic features such as desmin expression. ${ }^{19}$ Similarly, SM cells from the media of an injured blood vessel may lose late dif- ferentiation markers such as desmin, smoothelin, and SM myosin and acquire a myofibroblast phenotype. ${ }^{20}$

Lastly, myofibroblasts show both fibronexus junctions with other cells and specialized junctional complexes with the ECM; these large mature focal adhesions allow them to make strong attachments, contract and remodel the ECM, and provide a means of transducing mechanical force in the tissue. $^{21,22}$ The contractile nature of myofibroblasts has some similarities to SM cells, despite the differences in expression of cytoskeletal features. For example, the calcium signaling in myofibroblasts appears to be similar to that in SM cells, and the arrangement of cells into something resembling that of SM cells in tissues with single-unit SM is also similar, that is, with cells having junctional complexes and connections (including gap junctions) that allow the spread of contraction signals through the tissue. Contraction of myofibroblasts seems to be possible through $\mathrm{Ca}^{2+}$-dependent mechanisms that are similar to those present in SM cells, with increased free $\mathrm{Ca}^{2+}$ regulating phosphorylation of myosin light chain. This may explain the rapid contractile responses of myofibroblasts in vitro to agonists such as angiotensin II or endothelin. Slower and more sustained contraction, which is perhaps more typical of what occurs during slow retractile contraction of connective tissue in granulation tissue, involves activity of the guanosine triphosphate (GTP)-ase RhoA and activation of its downstream target Rho-associated kinase (ROCK). This results in more continued phosphorylation of myosin light chain and thus sustained contraction. ${ }^{23}$

\section{Role in wound healing}

Immediately after wounding, the healing process commences, leading to (partial) restoration of injured tissue. Wound healing proceeds in three interrelated dynamic phases that temporally overlap (Figure 1). Based on morphological changes over the course of the healing process, these phases are defined as the inflammatory phase, the proliferative phase (the development of granulation tissue), and the regeneration phase, including maturation, scar formation, and re-epithelialization. ${ }^{24}$ The inflammatory phase begins with damage of capillaries, triggering the formation of a blood clot consisting of fibrin and fibronectin. This provisional matrix fills in the lesion and allows a variety of recruited cells to migrate into the lesion. Platelets present in the blood clot release multiple chemokines, which participate in the recruitment of inflammatory cells, neutrophils, and macrophages, but also in chemotaxis and recruitment of fibroblasts and endothelial cells. The second stage of wound healing is the proliferative phase. Angiogenesis, which is 


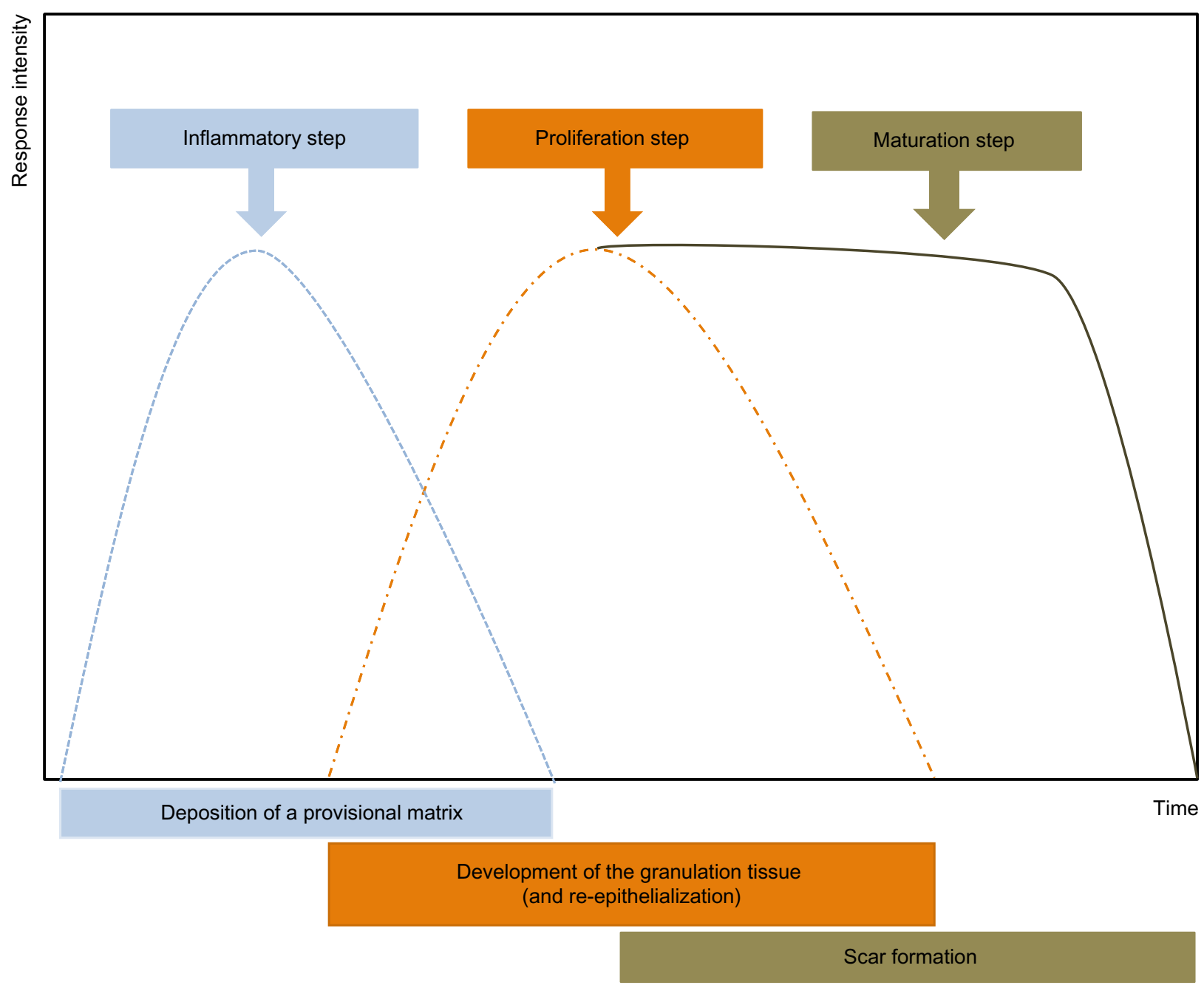

Figure I The various phases of the healing process.

Notes: After damage, inflammation leads to the formation of the granulation tissue, during which myofibroblasts appear. An important neoangiogenesis is also observed. On this granulation tissue, a new epidermis can then develop. Subsequently, remodeling of this granulation tissue occurs with apoptosis of the cells present in the granulation tissue (myofibroblasts and vascular cells) and reorganization of the extracellular matrix.

critical for the wound healing process, allows new capillaries to deliver nutrients to the wound, and contributes to the proliferation of fibroblasts. Initially the wound is hypoxic due to the loss of vascular perfusion, but with the development of a new capillary network, vascular perfusion is restored. Regulating wound angiogenesis in itself may represent a means for improving healing in some cases, particularly where delayed or defective angiogenesis is implicated in healing impairment. ${ }^{25}$ In the granulation tissue, fibroblasts are activated and acquire $\alpha$-SM actin expression and become myofibroblasts. These myofibroblastic cells synthesize and deposit the ECM components that eventually replace the provisional matrix (Figure 2). These cells exhibit contractile properties, due to the expression of $\alpha$-SM actin in microfilament bundles or stress fibers, playing a major role in the contraction and maturation of the granulation tissue. ${ }^{26}$
Presently, it is accepted that the myofibroblastic modulation of fibroblastic cells begins with the appearance of the protomyofibroblast, whose stress fibers contain only $\beta$ - and $\gamma$-cytoplasmic actins. Protomyofibroblasts generally evolve into differentiated myofibroblasts, the most common variant of this cell, with stress fibers containing $\alpha$-SM actin (for review, see Tomasek et $\mathrm{al}^{27}$ ). Myofibroblasts can, depending on the experimental or clinical situation, express other SM-related contractile proteins, such as SM myosin heavy chains or desmin; however, the presence of $\alpha$-SM actin represents the most reliable marker of the myofibroblastic phenotype. ${ }^{27}$ The third phase of healing, scar formation, involves a progressive remodeling of the granulation tissue. During this remodeling process, proteolytic enzymes, essentially matrix metalloproteinases (MMPs) and their inhibitors (tissue inhibitor of metalloproteinases [TIMPs]) play a major 


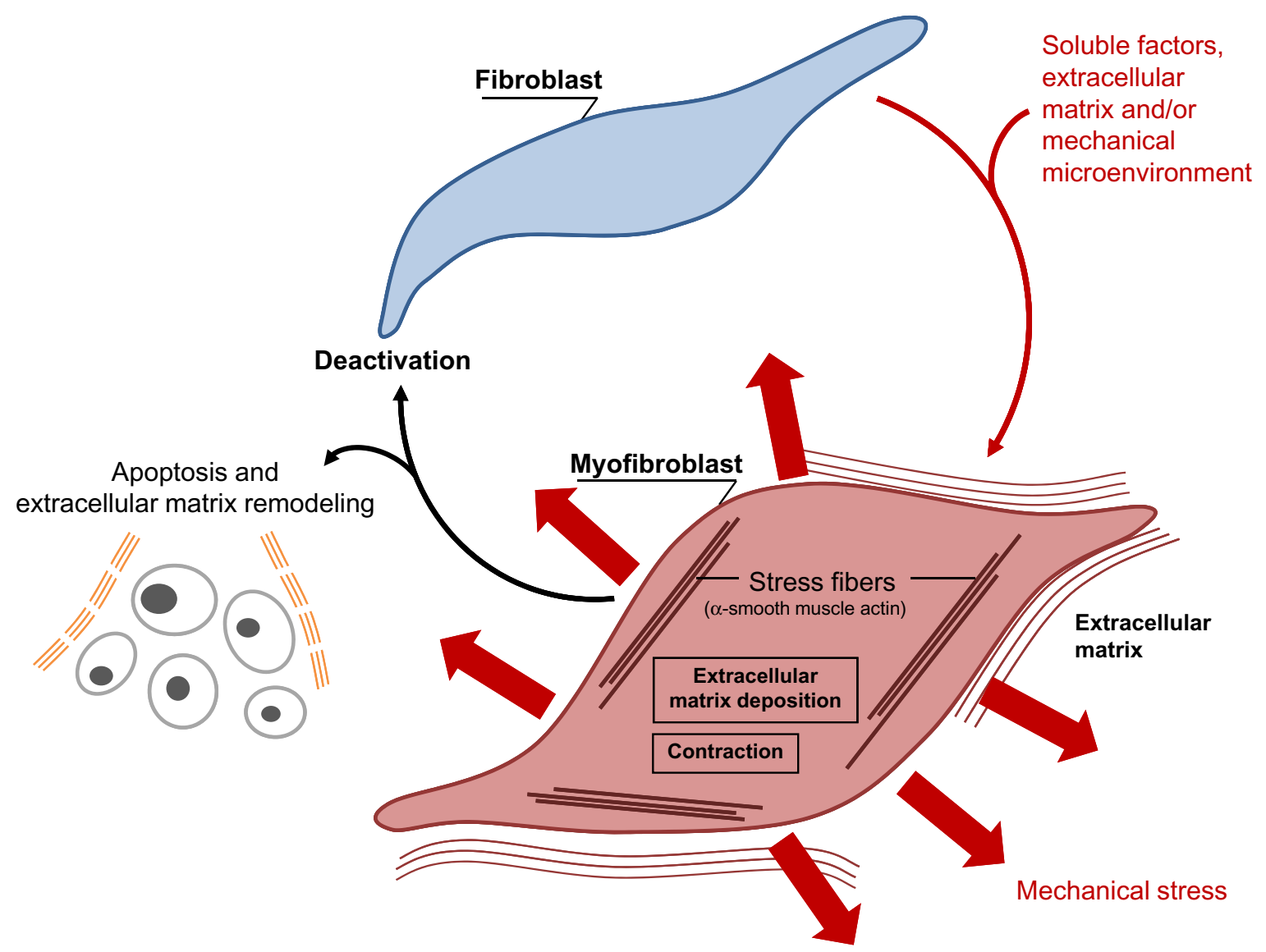

Figure 2 Schematic illustration showing the evolution of the (myo)fibroblast phenotype.

Notes: The myofibroblastic modulation of fibroblastic cells begins with the appearance of the proto myofibroblast, whose stress fibers contain only $\beta$ - and $\gamma$-cytoplasmic actins and evolves, but not necessarily always, into the appearance of the differentiated myofibroblast, the most common variant of this cell, with stress fibers containing $\alpha$-smooth muscle actin. Soluble factors, extracellular matrix components, and/or the mechanical microenvironment are involved in myofibroblastic differentiation. The myofibroblast can disappear by apoptosis; while deactivation leading to a quiescent phenotype has not been clearly demonstrated, at least in vivo.

role. ${ }^{28}$ The synthesis of ECM is not totally stopped, but considerably reduced, and the synthesized components are modified as the matrix is remodeled. Progressively, collagen type III, the major component of the granulation tissue, is replaced by collagen type $I$, which is the main structural component of the dermis. Lastly, elastin, which contributes to skin elasticity and is absent in the granulation tissue, also reappears. In the resolution phase of healing, the cell number is dramatically reduced by apoptosis of both vascular cells and myofibroblasts. ${ }^{29}$ To date, it is not known whether myofibroblasts can reacquire a quiescent phenotype, that is, return to a normal dermal fibroblast phenotype with no expression of $\alpha-\mathrm{SM}$ actin (Figure 2).

\section{Origin of wound myofibroblasts}

It is generally accepted that the major source of myofibroblasts are local connective tissue fibroblasts that are recruited into the wound. ${ }^{30}$ Dermal fibroblasts located at the edges of the wound can acquire a myofibroblastic phenotype and par- ticipate in tissue repair. ${ }^{31}$ However, important heterogeneity in fibroblastic cell subpopulations has also been observed. These subpopulations reside in different locations within the skin and have specific activation and deactivation properties. At least three subpopulations have been identified in the dermis: superficial (or papillary) fibroblasts (papillary dermis is around 300-400 $\mu \mathrm{m}$ deep and is arranged as a ridge-like structure), reticular fibroblasts, which reside in the deep dermis (made of thick collagen and elastin fibers arranged parallel to the surface of the skin), and fibroblasts associated with hair follicles. These cell subpopulations can be isolated and exhibit, depending of the nature and age of the original skin, distinct phenotypic differences when cultured separately. ${ }^{32,33}$

Recently, the involvement in tissue repair of local mesenchymal stem cells has been increasingly raised. These progenitor cells have been described in the dermal sheath that surrounds the outside of the hair follicle facing the epithelial stem cells. They are involved in the regeneration 
of the dermal papilla and can also became wound healing (myo)fibroblasts after a lesion or injury. ${ }^{34,35}$

Recent data have also implicated circulating cells, dubbed fibrocytes, in the tissue repair process. Fibrocytes enter injured skin together with inflammatory cells and may then acquire a myofibroblastic phenotype. ${ }^{36}$ In postburn scars, fibrocytes are recruited to the site of the lesion where they stimulate the local inflammatory response and produce ECM proteins, thus contributing to hypertrophic scar formation. ${ }^{37}$

Another type of circulating cell originating from bone marrow has also been suggested to play a role in tissue repair. Mesenchymal stem cells are bone marrow-derived non-hematopoietic precursor cells ${ }^{38}$ that contribute to the maintenance and regeneration of connective tissues through engraftment. ${ }^{39}$ Indeed, they have the capacity to seed into several organs and to differentiate into wound-healing myofibroblasts. This engraftment in injured organs is regulated by the severity of the damage. ${ }^{40}$

Finally, differentiated (or malignant) epithelial and endothelial cells can undergo a phenotypic conversion that gives rise to matrix-producing fibroblasts and myofibroblasts (through epithelial- and endothelial-to-mesenchymal transition processes). ${ }^{41}$ This mechanism is increasingly recognized as an integral part of tissue fibrogenesis after injury, but seems to play a limited role during normal tissue repair.

Overall, mesenchymal stem cells, fibrocytes, bone marrow-derived cells, and cells derived from epithelialand endothelial-to-mesenchymal transition processes may represent alternative sources of myofibroblasts when local fibroblasts are not sufficient to carry out tissue repair and remodeling.

\section{Role of myofibroblasts in diseases (excessive scarring/fibrosis)}

Myofibroblasts are implicated in many fibrotic and scarring diseases, where they carry out the important process after initial injury of providing mechanical support and integrity to the tissue. In normal physiological conditions, they are then lost via apoptosis, generally when the tissue integrity has been sufficiently restored to be mechanically coherent. ${ }^{9,29}$ Thus, in normal physiological situations like skin wound healing, myofibroblasts disappear in a prominent wave of apoptosis, leaving a markedly less cellular scar. However, it is now assumed that, in many fibrotic and scarring conditions, as well as in the stromal response to tumors, myofibroblasts fail to undergo cell death, persist, and thus in turn lead to ongoing pathology and scarring (Figure 3). An example of reduced or inhibited apoptosis leading to scarring is in a model of hypertrophic scarring, where mechanical loading increases survival of myofibroblasts and was found to lead to greater scar formation. ${ }^{42,43} \mathrm{~A}$ better understanding of the control and signaling that governs apoptosis (or autophagy) in myofibroblasts may lead to more targeted approaches to combatting fibrosis and scarring. It has been demonstrated that elevated nicotinamide adenine dinucleotide phosphate (NADPH) oxidase 4-derived hydrogen peroxide, supported by concomitant decreases in nitric oxide signaling and reactive oxygen species scavengers, are central factors in the molecular pathogenesis of fibrosis. ${ }^{44}$ Redox signaling could therefore represent an interesting target to restore the physiological fibroblast-myofibroblast ratio. Apoptosis in myofibroblasts is thought to be regulated by a reduction in the local growth factors that drive and sustain myofibroblast differentiation. In particular, local concentrations of transforming growth factor (TGF)- $\beta 1$ and endothelin-1 play a role in stimulating myofibroblast survival via protein kinase B (AKT) activation. ${ }^{45}$ However, changes in mechanical signaling such as unloading of mechanical force likely also plays a role (discussed below).

The importance of myofibroblasts in causing fibrosis in internal organs and the skin (hypertrophic scars), and the role that persistence of stromal myofibroblasts appear to play in tumor growth and spread, makes the (down)regulation of myofibroblasts and the potential regulation of myofibroblast disappearance through apoptosis of increasing interest (Figure 4) (for review, see Hinz and Gabbiani ${ }^{46}$ ).

\section{Regulation of myofibroblasts by mechanical forces}

Mechanical signals have been shown to play a role in myofibroblast differentiation as the ECM that surrounds the fibroblasts in damaged tissue changes its composition and its stiffness as tissue repair proceeds. ${ }^{47}$ The early ECM present in damaged tissue, or provisional matrix, is rich in fibrin and has been estimated to be very compliant. Fibroblasts cultured in compliant ECM such as soft three-dimensional (3D) collagen gels, show little development of stress fibers. These fibroblasts then form only small adhesions with the ECM. ${ }^{48,49}$ Fibroblasts grown in stiffer collagen matrices have been shown to form stress fibers and mature focal adhesions, though they are still negative for the myofibroblast marker $\alpha-S M$ actin. Lastly, the stiff matrix found either in 3D cultures using stiff (higher concentration) collagen matrix or in vivo in granulation tissue and fibrotic tissues is able to induce full myofibroblast differentiation in concert with 


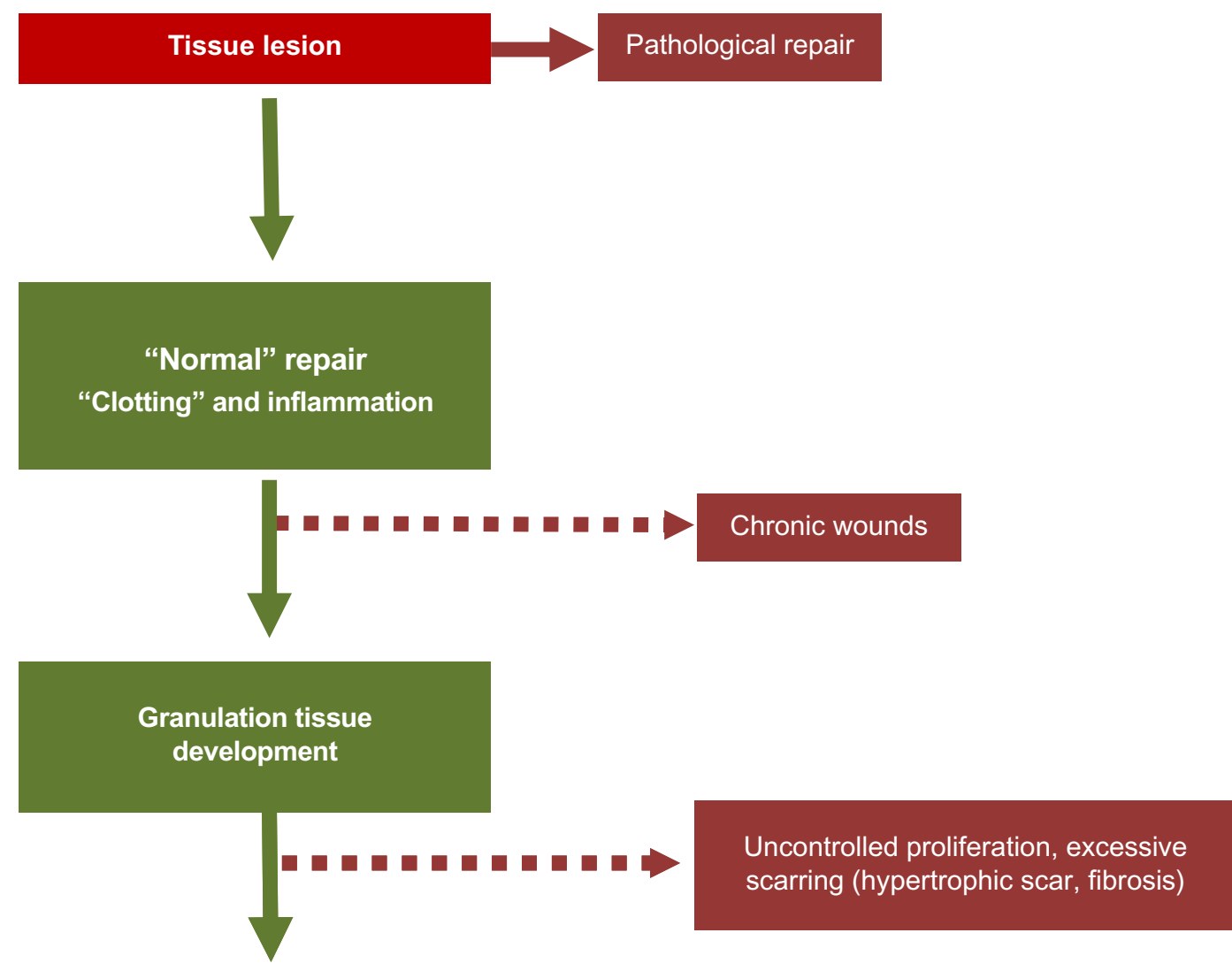

\section{Scar formation}

Figure 3 Pathological situations.

Notes: If the inflammation phase persists and the granulation tissue does not develop, a chronic wound may result. If the remodeling phase of the granulation tissue does not happen (neither apoptosis of the cells present in the granulation tissue, myofibroblasts, and vascular cells, nor reorganization of the extracellular matrix), myofibroblasts may persist, leading to pathological situations characterized by excessive scarring.

growth factor stimulation from TGF- $\beta 1 . .^{22}$ The contractile nature of myofibroblasts itself leads to an increase in stiffness and mechanical stress of the ECM as healing progresses, leading to a positive-feedback loop where increased stress signals myofibroblast differentiation and also increases myofibroblast survival. ${ }^{42}$ For this reason, mechanical feedback is considered to be important in driving pathological conditions such as contractures post-injury. The role of mechanical stress in stimulating myofibroblast activity has also been shown in experiments where dermal wounds in mice are mechanically stressed by stretching or splinting the wound, where increased myofibroblast activity results in increased scar formation, to some extent mimicking hypertrophic scarring that is seen in humans. ${ }^{42}$

In cancer biology, matrix stiffness can be used as a diagnostic indicator of the risk of malignancy and appears to correlate with increased invasiveness of tumors, for example in breast cancer where density of tissue on mammography correlates strongly with the risk of cancer formation. Recent studies have suggested this may be due to increased cell proliferation of epithelial and mesenchymal cells on stiffer matrices.

Conversely, releasing mechanical stress or reducing stiffness has been shown to induce both apoptosis and a reduction in $\alpha-\mathrm{SM}$ actin expression and contractility in myofibroblasts. ${ }^{50,51}$

Mechanical signaling and stress modulate myofibroblast differentiation via a number of pathways and mechanisms. Stress may directly activate transcription of the $\alpha$-SM actin gene, since application of force across integrin binding sites has been shown to up-regulate $\alpha$-SM actin promoter activity. ${ }^{52}$ As mentioned above, mechanical force alone is not generally sufficient to induce myofibroblast differentiation and other factors are needed to act in concert, specifically TGF- $\beta 1$. Mechanical signaling and TGF- $\beta 1$ stimulation also increase collagen gene expression by fibroblasts, emphasizing the role these factors play in stimulating a pro-fibrotic phenotype as is shown by activated myofibroblasts. TGF- $\beta 1$ 


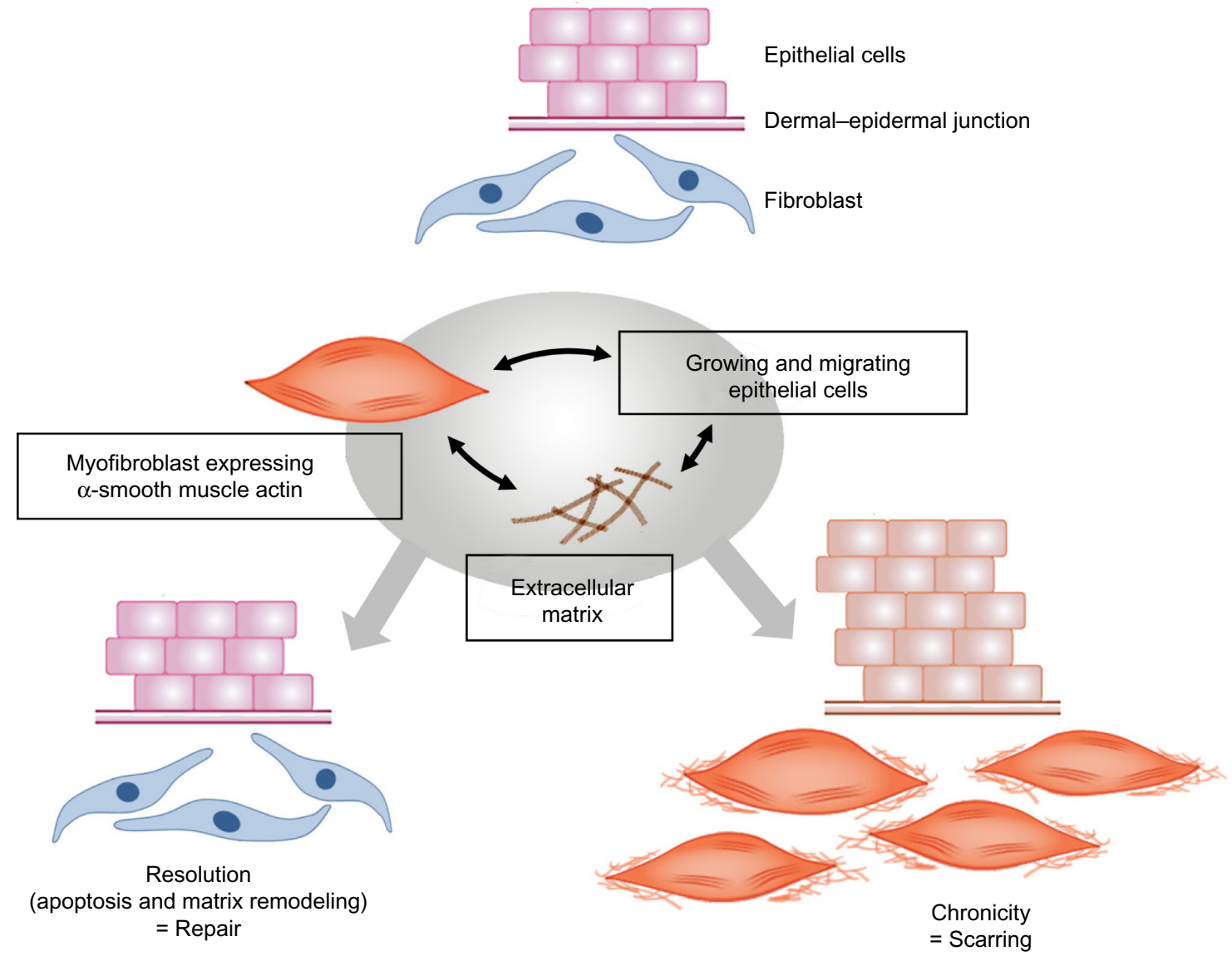

Figure 4 Processes leading to normal wound repair and pathological scarring.

Notes: In all of these situations, interactions between fibroblasts/myofibroblasts and the extracellular matrix, and also epithelial-mesenchymal cell dialogue, play a major role.

also favors deposition of ECM proteins over degradation by up-regulating TIMPs while decreasing expression of the MMPs themselves. ${ }^{53}$

Stimulation of myofibroblasts by TGF- $\beta 1$ itself is affected by mechanical forces within the damaged or fibrotic tissue. TGF- $\beta 1$ released from a variety of inflammatory cells and platelets in the microenvironment of damaged or fibrotic tissue is in a latent form. Indeed, myofibroblasts themselves release latent TGF- $\beta 1$ complexed with latency-associated peptide (LAP). Together with a binding protein, TGF- $\beta 1$ is bound to ECM proteins, providing a reservoir of latent TGF- $\beta 1$ that can be activated as healing and scar formation progress. ${ }^{54,55}$ Myofibroblasts express integrins that can bind to the LAP, and mechanical stress applied to the integrins, either by mechanical stress on the matrix and/or via myofibroblast contraction, can effectively activate TGF- $\beta 1$ without cleaving the LAP and allow its binding to cell membrane receptors. ${ }^{51}$ Thus, both increased mechanical stress and contraction can further increase myofibroblast contractile and matrix synthetic activity. This mode of activation provides another possible pathway for regulating myofibroblast activity by blocking integrin binding to latent TGF- $\beta 1$, for example by blocking the integrin involved in latent TGF- $\beta 1$ activation, $\alpha v \beta 5 .^{56}$ Inhibition of other integrin-binding sites has also been shown to inhibit myofibroblast development, including blocking of integrins $\alpha 3 \beta 1,{ }^{57} \alpha 11 \beta 1,{ }^{58} \alpha v \beta 5,{ }^{59}$ or $\beta 1 .{ }^{60}$

\section{Hypoxia}

Tissue oxygenation or hypoxia may play a role in both normal healing and pathological situations. In normal wound healing, the wound is transiently hypoxic as vascular perfusion is disrupted by the initial injury. Staining for the hypoxia-induced transcription factor, hypoxia inducible factor (HIF)- $1 \alpha$ shows both areas of the early granulation tissue and the overlying migrating keratinocytes to be hypoxic. During normal healing, this hypoxia is resolved within a few days of injury and expression of HIF- $1 \alpha$ declines. Hypoxia signaling can induce a number of growth factors that are beneficial to the healing process, prominent amongst them being vascular endothelial growth factor (VEGF) and, thus, acute hypoxia likely plays a beneficial role in healing. However, the same may not be true for chronic hypoxia and chronic hypoxia signaling. 
Hypoxia has been reported to reduce myofibroblast activation and reduce collagen synthesis and $\alpha$-SM actin expression. ${ }^{61}$ In vivo studies using HIF-1-deficient mice showed that reducing HIF-1 availability during healing resulted in reduced collagen synthesis and delayed myofibroblast differentiation, suggesting that, overall, in vivo acute hypoxia during healing was normally compensated by induction of genes that allow tissue to adapt to transient hypoxia, such as VEGF. ${ }^{62}$ In fact, fibroblasts that show reduced HIF-1 $\alpha$ expression during hypoxia show inhibited migration and collagen synthesis in vitro. It is possible that, in some organs where there is pathological fibrosis and scarring, hypoxia and HIF signaling, possibly during more chronic hypoxic states, actually drives fibrosis, which has been suggested in the case of renal fibrosis. In some cells at least, there is cross talk between hypoxia signaling and TGF- $\beta$ signaling that may exacerbate matrix synthesis and thus fibrosis. ${ }^{63,64}$

\section{Therapies}

Anti-fibrotic and anti-scarring therapies have proven to be a difficult and elusive area for research, with relatively few advances until quite recently. As the growth factor TGF- $\beta$ is central to many of the mechanisms of pathological scarring and fibrosis, it has been the target of some therapeutic strategies. Some positive results have been reported with the drug pirfenidone, particularly in lung fibrosis, where the drug lowered TGF- $\beta$ expression and both tissue and lavage fluid levels of the protein. ${ }^{65}$ Interfering with activation of latent TGF- $\beta$ is another potential target for anti-scarring therapies, and the role of integrin binding in TGF- $\beta$ activation makes integrin-blocking antibodies a potential therapy for lowering TGF- $\beta$ activation and thus downstream signaling. ${ }^{66,67}$ Specific inhibitors of TGF- $\beta$ signaling have also been suggested as possible treatments for scarring and fibrosis. TGF- $\beta$ exerts its pro-fibrotic effects through transcription factor signaling Smad3, and selective inhibition of Smad3 phosphorylation and inhibition of Smad3 interaction with Smad4 has been shown to reduce fibroblast activation to the myofibroblast phenotype and also reduce ECM synthetic activity of the cells. ${ }^{68}$ Other molecular targets include tyrosine kinases, and the drug imatinib mesylate has been reported to be antifibrotic through inhibiting downstream molecules that are required for the TGF- $\beta$ response while having an additional anti-fibrotic role by also inhibiting platelet-derived growth

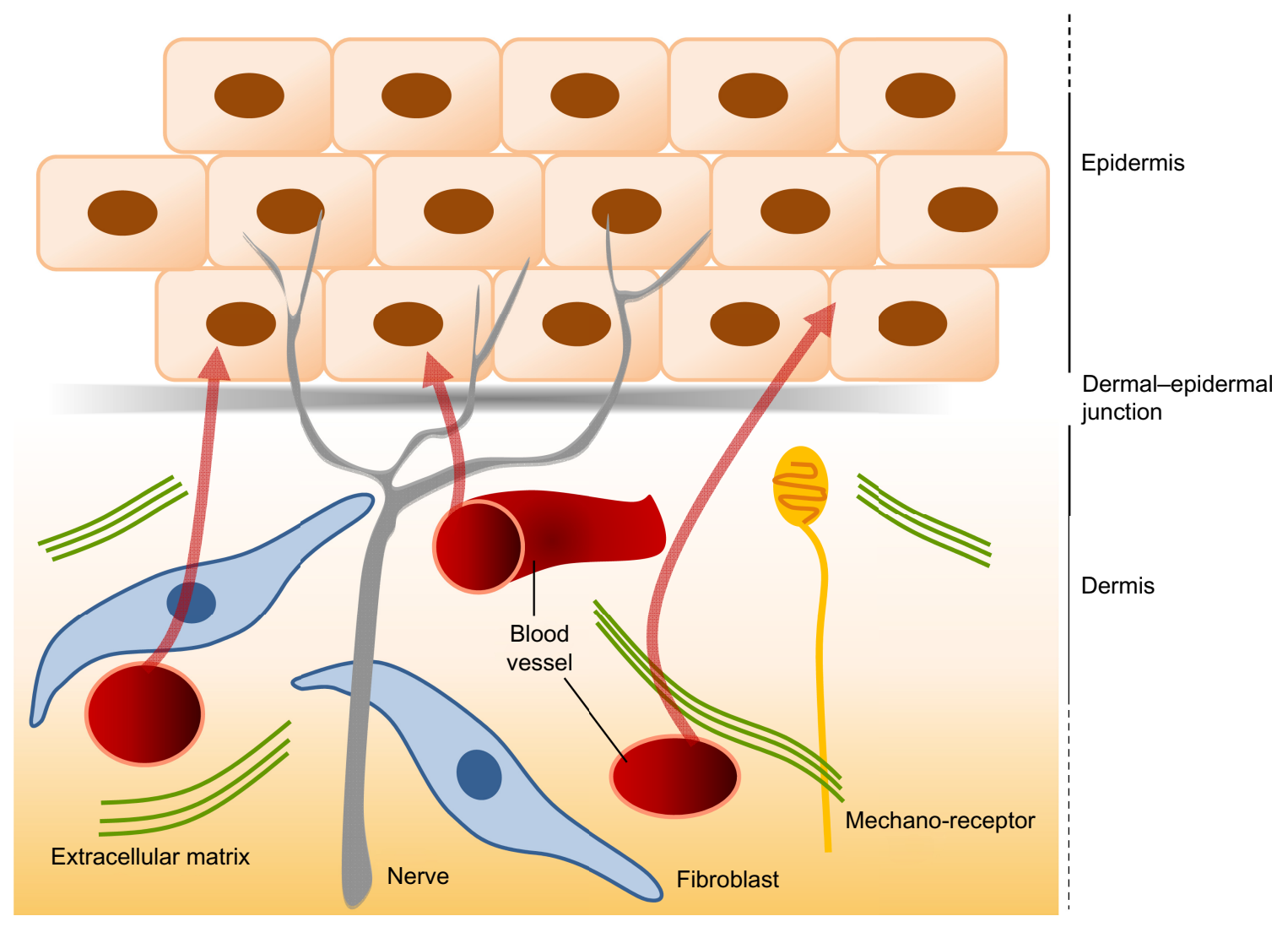

Figure $\mathbf{5}$ The interactions between the dermis and the epidermis.

Notes: The dermis and the epidermis contain nerves, but only the dermis is vascularized. The epidermis thus derives all its nutrients from dermal vessels (arrows). The dermalepidermal junction plays a major role in the intense dialogue that exists between the keratinocytes of the epidermis and the cells of the dermis, notably the fibroblasts. 
factor signaling. ${ }^{69}$ Interestingly, HMG-coA reductase inhibitors such as statins have been shown to have anti-fibrotic effects, likely through inhibition of ROCK. ${ }^{70}$ The widespread use of, and low rate of side effects associated with, these drugs may make them promising as anti-fibrotic therapies in the future.

\section{Conclusion}

Since their first description in the early 1970s, our knowledge about myofibroblast biology has increased greatly, and our interest in the biology of myofibroblasts has also increased, as this cell has been implicated in many pathological situations in addition to their role in normal wound repair. Despite major advances in our understanding of the origins of myofibroblasts and the factors that regulate their differentiation and activity, it remains a challenge and a major goal of researchers to find ways in which to regulate their activity to improve healing and scarring in the clinic. It is interesting that the skin is a highly sensitive organ. It is densely innervated by different sensory nerve fiber subtypes that react to tissue injury, temperature variation, and tactile stimuli (Figure 5). Cutaneous sensory nerve fibers are endings of dorsal root ganglia (or spinal ganglia) neurons that carry signals from sensory organs toward the appropriate integration center of the brain or of the spinal cord. Several clinical observations indicate that damage to the peripheral nervous system influences wound healing, sometimes resulting in chronic wounds within the affected area. Patients with cutaneous sensory defects due to spinal cord injury or diabetic neuropathy have an increased risk of developing ulcers that fail to heal. In addition, in aged patients, cutaneous repair processes are less efficient and this could be partly due to a deterioration of the peripheral nervous system at the skin level. Interestingly, factors that are required for sustaining peripheral nerves, the neurotrophin network, have also been shown to have direct effects on dermal fibroblasts in regulating ECM secretion, fibroblast differentiation, and tensile strength via effects on myofibroblasts. ${ }^{71}$ Understanding the role of innervation during wound healing and myofibroblastic differentiation therefore represents an interesting domain. In addition, cutaneous innervation is certainly necessary to provide good hemostasis and to maintain the mechanical and cosmetic properties of the skin. ${ }^{72}$ In conclusion, the recent advances made in understanding control of differentiation, proliferation, and survival of myofibroblasts will hopefully lead to new therapeutic approaches to limit scarring and improve healing in the not-too-distant future. ${ }^{73}$

\section{Acknowledgments}

This work was supported in part by the French Armaments Procurement Agency (DGA, No 201394 0903). Betty Laverdet was recipient of a fellowship from the French Armaments Procurement Agency. The authors thank Régine Baudet (LVMH Recherche) for the production of the figures.

\section{Disclosure}

The authors report no conflicts of interest directly relevant to the content of this paper.

\section{References}

1. Gabbiani G, Ryan GB, Majno G. Presence of modified fibroblasts in granulation tissue and their possible role in wound contraction. Experientia. 1971;27(5):549-550.

2. Desmoulière A, Darby IA, Gabbiani G. Normal and pathologic soft tissue remodeling: role of the myofibroblast, with special emphasis on liver and kidney fibrosis. Lab Invest. 2003;83(12):1689-1707.

3. Eyden B. The myofibroblast: a study of normal, reactive and neoplastic tissues, with an emphasis on ultrastructure. part 2 - tumours and tumour-like lesions. J Submicrosc Cytol Pathol. 2005;37(3-4): 231-296.

4. Darby IA, Hewitson TD. Fibroblast differentiation in wound healing and fibrosis. Int Rev Cytol. 2007;257:143-179.

5. Desmoulière A, Guyot C, Gabbiani G. The stroma reaction myofibroblast: a key player in the control of tumor cell behavior. Int $J$ Dev Biol. 2004;48(5-6):509-517.

6. Kalluri R, Zeisberg M. Fibroblasts in cancer. Nat Rev Cancer. 2006; 6(5):392-401.

7. De Wever O, Demetter P, Mareel M, Bracke M. Stromal myofibroblasts are drivers of invasive cancer growth. Int $J$ Cancer. 2008;123(10): 2229-2238.

8. Tsujino T, Seshimo I, Yamamoto H, et al. Stromal myofibroblasts predict disease recurrence for colorectal cancer. Clin Cancer Res. 2007;13(7):2082-2090.

9. Darby I, Skalli O, Gabbiani G. Alpha-smooth muscle actin is transiently expressed by myofibroblasts during experimental wound healing. Lab Invest. 1990;63(1):21-29.

10. Hinz B. Formation and function of the myofibroblast during tissue repair. J Invest Dermatol. 2007;127(3):526-537.

11. Sandbo N, Dulin N. Actin cytoskeleton in myofibroblast differentiation: ultrastructure defining form and driving function. Transl Res. 2011; 158(4):181-196.

12. Serini G, Bochaton-Piallat ML, Ropraz P, et al. The fibronectin domain ED-A is crucial for myofibroblastic phenotype induction by transforming growth factor-beta1. J Cell Biol. 1998;142(3):873-881.

13. Hinz B, Pittet P, Smith-Clerc J, Chaponnier C, Meister JJ. Myofibroblast development is characterized by specific cell-cell adherens junctions. Mol Biol Cell. 2004;15(9):4310-4320.

14. Benzonana G, Skalli O, Gabbiani G. Correlation between the distribution of smooth muscle or non muscle myosins and alpha-smooth muscle actin in normal and pathological soft tissues. Cell Motil Cytoskeleton. 1988;11(4):260-274.

15. Eyden B. The myofibroblast: a study of normal, reactive and neoplastic tissues, with an emphasis on ultrastructure. J Submicrosc Cytol Pathol. 2007:7-166.

16. van der Loop FT, Schaart G, Timmer ED, Ramaekers FC, van Eys GJ. Smoothelin, a novel cytoskeletal protein specific for smooth muscle cells. J Cell Biol. 1996;134(2):401-411.

17. Hinz B, Mastrangelo D, Iselin CE, Chaponnier C, Gabbiani G. Mechanical tension controls granulation tissue contractile activity and myofibroblast differentiation. Am J Pathol. 2001;159(3):1009-1020. 
18. Armulik A, Abramsson A, Betsholtz C. Endothelial/pericyte interactions. Circ Res. 2005;97(6):512-523.

19. Rajkumar VS, Shiwen X, Bostrom M, et al. Platelet-derived growth factor-beta receptor activation is essential for fibroblast and pericyte recruitment during cutaneous wound healing. Am J Pathol. 2006;169(6): 2254-2265.

20. Hao H, Gabbiani G, Camenzind E, Bacchetta M, Virmani R, BochatonPiallat ML. Phenotypic modulation of intima and media smooth muscle cells in fatal cases of coronary artery lesion. Arterioscler Thromb Vasc Biol. 2006;26(2):326-332.

21. Dugina V, Fontao L, Chaponnier C, Vasiliev J, Gabbiani G. Focal adhesion features during myofibroblastic differentiation are controlled by intracellular and extracellular factors. $J$ Cell Sci. 2001;114(Pt 18): 3285-3296.

22. Goffin JM, Pittet P, Csucs G, Lussi JW, Meister JJ, Hinz B. Focal adhesion size controls tension-dependent recruitment of alpha-smooth muscle actin to stress fibers. J Cell Biol. 2006;172(2):259-268.

23. Follonier Castella L, Gabbiani G, McCulloch CA, Hinz B. Regulation of myofibroblast activities: calcium pulls some strings behind the scene. Exp Cell Res. 2010;316(15):2390-2401.

24. Singer AJ, Clark RA. Cutaneous wound healing. $N$ Engl J Med. 1999;341(10):738-746.

25. Wietecha MS, Dipietro LA. Therapeutic approaches to the regulation of wound angiogenesis. Adv Wound Care (New Rochelle). 2013;2(3): $81-86$.

26. Hinz B, Gabbiani G. Cell-matrix and cell-cell contacts of myofibroblasts: role in connective tissue remodeling. Thromb Haemost. 2003; 90(6):993-1002.

27. Tomasek JJ, Gabbiani G, Hinz B, Chaponnier C, Brown RA. Myofibroblasts and mechano-regulation of connective tissue remodelling. Nat Rev Mol Cell Biol. 2002;3(5):349-363.

28. Visse R, Nagase H. Matrix metalloproteinases and tissue inhibitors of metalloproteinases: structure, function, and biochemistry. Circ Res. 2003;92(8):827-839.

29. Desmoulière A, Redard M, Darby I, Gabbiani G. Apoptosis mediates the decrease in cellularity during the transition between granulation tissue and scar. Am J Pathol. 1995;146(1):56-66.

30. Higashiyama R, Nakao S, Shibusawa Y, et al. Differential contribution of dermal resident and bone marrow-derived cells to collagen production during wound healing and fibrogenesis in mice. J Invest Dermatol. 2011;131(2):529-536.

31. Driskell RR, Lichtenberger BM, Hoste E, et al. Distinct fibroblast lineages determine dermal architecture in skin development and repair. Nature. 2013;504(7479):277-281.

32. Sorrell JM, Caplan AI. Fibroblast heterogeneity: more than skin deep. J Cell Sci. 2004;117(Pt 5):667-675.

33. Mine S, Fortunel NO, Pageon H, Asselineau D. Aging alters functionally human dermal papillary fibroblasts but not reticular fibroblasts: a new view of skin morphogenesis and aging. PLoS One. 2008;3(12):e4066.

34. Jahoda CA, Reynolds AJ. Hair follicle dermal sheath cells: unsung participants in wound healing. Lancet. 2001;358(9291):1445-1448.

35. Jahoda CA, Whitehouse J, Reynolds AJ, Hole N. Hair follicle dermal cells differentiate into adipogenic and osteogenic lineages. Exp Dermatol. 2003;12(6):849-859.

36. Abe R, Donnelly SC, Peng T, Bucala R, Metz CN. Peripheral blood fibrocytes: differentiation pathway and migration to wound sites. J Immunol. 2001;166(12):7556-7562.

37. Yang L, Scott PG, Dodd C, et al. Identification of fibrocytes in postburn hypertrophic scar. Wound Repair Regen. 2005;13(4):398-404.

38. Pittenger MF, Mackay AM, Beck SC, et al. Multilineage potential of adult human mesenchymal stem cells. Science. 1999;284(5411):143-147.

39. Opalenik SR, Davidson JM. Fibroblast differentiation of bone marrow-derived cells during wound repair. FASEB J. 2005;19(11): $1561-1563$.

40. Direkze NC, Forbes SJ, Brittan M, et al. Multiple organ engraftment by bone-marrow-derived myofibroblasts and fibroblasts in bone-marrow-transplanted mice. Stem Cells. 2003;21(5):514-520.
41. Nakamura M, Tokura Y. Epithelial-mesenchymal transition in the skin. J Dermatol Sci. 2011;61(1):7-13.

42. Aarabi S, Bhatt KA, Shi Y, et al. Mechanical load initiates hypertrophic scar formation through decreased cellular apoptosis. FASEB J. 2007;21(12):3250-3261.

43. van der Veer WM, Bloemen MC, Ulrich MM, et al. Potential cellular and molecular causes of hypertrophic scar formation. Burns. 2009; 35(1):15-29.

44. Sampson N, Berger P, Zenzmaier C. Redox signaling as a therapeutic target to inhibit myofibroblast activation in degenerative fibrotic disease. Biomed Res Int. 2014;2014:131737.

45. Kulasekaran P, Scavone CA, Rogers DS, Arenberg DA, Thannickal VJ, Horowitz JC. Endothelin-1 and transforming growth factor-beta1 independently induce fibroblast resistance to apoptosis via AKT activation. Am J Respir Cell Mol Biol. 2009;41(4):484-493.

46. Hinz B, Gabbiani G. Fibrosis: recent advances in myofibroblast biology and new therapeutic perspectives. F1000 Biol Rep. 2010;2:78.

47. Hinz B. The myofibroblast: paradigm for a mechanically active cell. J Biomech. 2010;43(1):146-155.

48. Tamariz E, Grinnell F. Modulation of fibroblast morphology and adhesion during collagen matrix remodeling. Mol Biol Cell. 2002;13(11): 3915-3929.

49. Yeung T, Georges PC, Flanagan LA, et al. Effects of substrate stiffness on cell morphology, cytoskeletal structure, and adhesion. Cell Motil Cytoskeleton. 2005;60(1):24-34.

50. Grinnell F, Ho CH, Lin YC, Skuta G. Differences in the regulation of fibroblast contraction of floating versus stressed collagen matrices. J Biol Chem. 1999;274(2):918-923.

51. Wipff PJ, Rifkin DB, Meister JJ, Hinz B. Myofibroblast contraction activates latent TGF-beta1 from the extracellular matrix. $J$ Cell Biol. 2007;179(6):1311-1323.

52. Wang J, Chen H, Seth A, McCulloch CA. Mechanical force regulation of myofibroblast differentiation in cardiac fibroblasts. Am J Physiol Heart Circ Physiol. 2003;285(5):H1871-H1881.

53. Leask A, Abraham DJ. TGF-beta signaling and the fibrotic response. FASEB J. 2004;18(7):816-827.

54. Annes JP, Chen Y, Munger JS, Rifkin DB. Integrin alphaVbeta6mediated activation of latent TGF-beta requires the latent TGF-beta binding protein-1. J Cell Biol. 2004;165(5):723-734.

55. Buscemi L, Ramonet D, Klingberg F, et al. The single-molecule mechanics of the latent TGF- $\beta 1$ complex. Curr Biol. 2011;21(24): 2046-2054.

56. Zhou Y, Hagood JS, Lu B, Merryman WD, Murphy-Ullrich JE. Thy-1-integrin alphav beta5 interactions inhibit lung fibroblast contraction-induced latent transforming growth factor-beta1 activation and myofibroblast differentiation. J Biol Chem. 2010;285(29): 22382-22393.

57. Kim KK, Wei Y, Szekeres C, et al. Epithelial cell alpha3betal integrin links beta-catenin and Smad signaling to promote myofibroblast formation and pulmonary fibrosis. J Clin Invest. 2009;119(1):213-224.

58. Carracedo S, Lu N, Popova SN, Jonsson R, Eckes B, Gullberg D. The fibroblast integrin alpha1 1beta1 is induced in a mechanosensitive manner involving activin A and regulates myofibroblast differentiation. J Biol Chem. 2010;285(14):10434-10445.

59. Asano Y, Ihn H, Yamane K, Jinnin M, Tamaki K. Increased expression of integrin alphavbeta5 induces the myofibroblastic differentiation of dermal fibroblasts. Am J Pathol. 2006;168(2):499-510.

60. Chan MW, Chaudary F, Lee W, Copeland JW, McCulloch CA. Force-induced myofibroblast differentiation through collagen receptors is dependent on mammalian diaphanous (mDia). J Biol Chem. 2010;285(12):9273-9281.

61. Modarressi A, Pietramaggiori G, Godbout C, Vigato E, Pittet B, Hinz B. Hypoxia impairs skin myofibroblast differentiation and function. J Invest Dermatol. 2010;130(12):2818-2827.

62. Musyoka JN, Liu MC, Pouniotis DS, et al. Siah2-deficient mice show impaired skin wound repair. Wound Repair Regen. 2013;21(3): 437-447. 
63. Higgins DF, Kimura K, Bernhardt WM, et al. Hypoxia promotes fibrogenesis in vivo via HIF-1 stimulation of epithelial-to-mesenchymal transition. J Clin Invest. 2007;117(12):3810-3820.

64. Basu RK, Hubchak S, Hayashida T, Runyan CE, Schumacker PT, Schnaper HW. Interdependence of HIF- $1 \alpha$ and TGF- $\beta / \mathrm{Smad} 3$ signaling in normoxic and hypoxic renal epithelial cell collagen expression. Am J Physiol Renal Physiol. 2011;300(4):F898-F905.

65. Iyer SN, Gurujeyalakshmi G, Giri SN. Effects of pirfenidone on transforming growth factor-beta gene expression at the transcriptional level in bleomycin hamster model of lung fibrosis. J Pharmacol Exp Ther. 1999;291(1):367-373

66. Wipff PJ, Hinz B. Integrins and the activation of latent transforming growth factor betal - an intimate relationship. Eur J Cell Biol. 2008;87(8-9):601-615.

67. Puthawala K, Hadjiangelis N, Jacoby SC, et al. Inhibition of integrin alpha(v)beta6, an activator of latent transforming growth factor-beta, prevents radiation-induced lung fibrosis. Am J Respir Crit Care Med. 2008;177(1):82-90.
68. Jinnin M, Ihn H, Tamaki K. Characterization of SIS3, a novel specific inhibitor of Smad3, and its effect on transforming growth factor-beta1-induced extracellular matrix expression. Mol Pharmacol. 2006;69(2):597-607.

69. Distler JH, Jüngel A, Huber LC, et al. Imatinib mesylate reduces production of extracellular matrix and prevents development of experimental dermal fibrosis. Arthritis Rheum. 2007;56(1):311-322.

70. Louneva N, Huaman G, Fertala J, Jiménez SA. Inhibition of systemic sclerosis dermal fibroblast type I collagen production and gene expression by simvastatin. Arthritis Rheum. 2006;54(4):1298-1308.

71. Palazzo E, Marconi A, Truzzi F, et al. Role of neurotrophins on dermal fibroblast survival and differentiation. J Cell Physiol. 2012;227(3): 1017-1025.

72. Roosterman D, Goerge T, Schneider SW, Bunnett NW, Steinhoff M. Neuronal control of skin function: the skin as a neuroimmunoendocrine organ. Physiol Rev. 2006;86(4):1309-1379.

73. Hinz B, Phan SH, Thannickal VJ, et al. Recent developments in myofibroblast biology: paradigms for connective tissue remodeling. Am J Pathol. 2012;180(4):1340-1355.
Clinical, Cosmetic and Investigational Dermatology

\section{Publish your work in this journal}

Clinical, Cosmetic and Investigational Dermatology is an international, peer-reviewed, open access, online journal that focuses on the latest clinical and experimental research in all aspects of skin disease and cosmetic interventions. All areas of dermatology will be covered; contributions will be welcomed from all clinicians and

\section{Dovepress}

basic science researchers globally. This journal is indexed on CAS The manuscript management system is completely online and includes a very quick and fair peer-review system, which is all easy to use. Visit http://www.dovepress.com/testimonials.php to read real quotes from published authors. 